\title{
Knockdown of TNFAIP1 inhibits growth and induces apoptosis in osteosarcoma cells through inhibition of the nuclear factor- $\kappa B$ pathway
}

\author{
CHENG-LIN ZHANG $^{1 *}$, CE WANG $^{1 *}$, WANG-JUN YAN ${ }^{1}$, RUI GAO $^{1}$, YONG-HUA LI $^{2}$ and XU-HUI ZHOU ${ }^{1}$ \\ Departments of ${ }^{1}$ Orthopedic Surgery and ${ }^{2}$ Anesthesiology, Changzheng Hospital, \\ Second Military Medical University, Shanghai 200003, P.R. China
}

Received March 10, 2014; Accepted May 30, 2014

DOI: 10.3892/or.2014.3291

\begin{abstract}
Tumor necrosis factor- $\alpha$-inducible protein-1 (TNFAIP1) plays a role in DNA synthesis, DNA repair, cell apoptosis and human diseases including cancer, and may be involved in tumor progression and metastases. However, little is known concerning the function of TNFAIP1 in human osteosarcoma (OS). The aim of the present study was to investigate the function and underlying mechanisms of TNFAIP1 in human OS. The expression of TNFAIP1 was examined by immunohistochemical assay using a tissue microarray procedure. A loss-of-function experiment was performed to explore the effects of lentiviral-mediated TNFAIP1 siRNA (siTNFAIP1) on cell proliferation, invasive potential and apoptosis by MTT and Transwell assays and flow cytometric analysis in OS (MG-63 and U-2 OS) cells. The results showed that the expression of TNFAIP1 protein was significantly increased in OS tissues compared with that in adjacent noncancerous tissues (ANCTs) (73.3 vs. $48.9 \%, \mathrm{P}=0.018)$, and was correlated with the distant metastasis of the patients with OS $(\mathrm{P}=0.029)$. Knockdown of TNFAIP1 suppressed cell proliferation and invasion, and induced cell apoptosis in the OS cells together with the downregulation of p65 nuclear factor- $\kappa \mathrm{B}$ $(\mathrm{NF}-\mathrm{\kappa B})$, proliferating cell nuclear antigen (PCNA) and matrix metalloproteinase-2 (MMP-2) and upregulation of caspase-3. Collectively, our findings indicate that high expression of TNFAIP1 is associated with distant metastasis of OS, and
\end{abstract}

Correspondence to: Professor Xu-Hui Zhou, Department of Orthopedic Surgery, Changzheng Hospital, Second Military Medical University, 415 Fengyang Road, Shanghai 200003, P.R. China E-mail: zhouxuhui2014033@163.com

Dr Yong-Hua Li, Department of Anesthesiology, Changzheng Hospital, Second Military Medical University, 415 Fengyang Road, Shanghai 200003, P.R. China

E-mail: liyonghua201403@163.com

${ }^{*}$ Contributed equally

Key words: TNFAIP1, osteosarcoma, growth, apoptosis knockdown of TNFAIP1 inhibits the growth and invasion, and induces apoptosis in OS cells through inhibition of the NF- $\kappa \mathrm{B}$ pathway, suggesting that TNFAIP1 may act as a potential therapeutic target for the treatment of cancer.

\section{Introduction}

Osteosarcoma (OS) is a primary malignant bone tumor mostly occurring in adolescents and young adults with a second peak at middle age (1). OS patients are generally treated with high doses of neoadjuvant chemotherapy to prevent the outgrowth of micrometastases (2). However, $45 \%$ of OS patients develop distant metastases, leading to the death of OS patients (3). OS is also a genetic disease, and single or multiple mutations in genes related to growth and metastasis form the molecular genetic basis of malignant transformation and tumor progression (4). Thus, identification of molecular targets that can be exploited in the clinic to treat metastatic disease is desperately needed.

Tumor necrosis factor- $\alpha$-inducible protein-1 (TNFAIP1) is a protein which can be induced by tumor necrosis factor $\alpha$ (TNF $\alpha$ ) and interleukin-6 (IL-6), and may play roles in DNA synthesis, cell apoptosis and human diseases (5). TNFAIP1 has been identified to be highly expressed in Alzheimer's disease brains (6) and hepatitis B virus (7), and is associated with diabetic nephropathy (8). Moreover, overexpression of TNFAIP1 was found to correlate with histological grade and a poor prognosis in breast cancer (9), and its allelic partner TNFAIP2 was found to be aberrantly expressed in malignant cells of classical Hodgkin lymphoma and primary mediastinal large B-cell lymphoma (10), and contributes to the rapid progression of cervical cancer (11), indicating TNFAIP may serve as a useful new marker of cancer.

TNFAIP1 expression was found to be downregulated in lipopolysaccharide (LPS)-induced liver injury (12). Yet, few reports have demonstrated the expression and function of TNFAIP1 in OS tissues. More importantly, TNFRSF11A gene encoding receptor activator of nuclear factor- $\kappa \mathrm{B}(\mathrm{NF}-\kappa \mathrm{B})$ has been confirmed to be responsible for the progression of OS (13), and activation of $\mathrm{NF}-\kappa \mathrm{B}$ participates in the antiapoptotic effect in TNF $\alpha$-treated Ewing sarcoma cells (14). In the present study, the expression of TNFAIP1 was examined, 
and its function and molecular mechanisms were evaluated in human OS. We found that knockdown of TNFAIP1 inhibited the growth and invasion, and induced apoptosis in OS cells through inhibition of the $\mathrm{NF}-\kappa \mathrm{B}$ pathway.

\section{Materials and methods}

Materials. The OS cell lines (MG-63 and U-2 OS) used for experiments were obtained from the Institute of Biochemistry and Cell Biology (Shanghai, China). Lentiviral-mediated TNFAIP1 siRNA (siTNFAIP1), the negative control (NC) vector, and virion-packaging elements were purchased from GeneChem (Shanghai, China). Human OS tissues and the corresponding adjacent non-cancerous tissues (ANCTs) were collected from the Department of Orthopedic Surgery, Changzheng Hospital. The OS tissue microarray was constructed by Shanghai Outdo Biotech Co. Ltd. (Shanghai, China). All the antibodies were purchased from Cell Signaling Technology (Boston, MA, USA). TNFAIP1 primer was synthesized by ABI (Framingham, MA, USA).

Drugs and reagents. Dulbecco's modified Eagle's medium (DMEM) and fetal bovine serum (FBS) were purchased from Thermo Fisher Scientific Inc. (Waltham, MA, USA); TRIzol reagent and Lipofectamine 2000 were obtained from Invitrogen (Carlsbad, CA, USA); M-MLV reverse transcriptase was purchased from Promega (Madison, WI, USA); SYBR-Green Master Mix was obtained from Takara (Otsu, Japan); and the ECL Plus kit was obtained from GE Healthcare (Piscataway, NJ, USA). The cell apoptosis kit was from KeyGen Biology (China).

Clinical samples and data. The tissue microarray was prepared for immunohistochemical (IHC) analysis. Human OS tissues and the corresponding ANCTs were obtained from biopsies in a total of 45 consecutive OS cases admitted to our hospital from January 2005 to December 2011. The baseline characteristics of the patients before neoadjuvant chemotherapy are summarized in Table I. The present study was approved by the Medical Ethics Committee of the Second Military Medical University, and written informed consent was obtained from the patients or their parents before sample collection. Two pathologists respectively reviewed all of the cases.

Tissue microarray. The Advanced tissue arrayer (ATA-100; Chemicon International, Tamecula, CA, USA) was used to create holes in a recipient paraffin block and to acquire cylindrical core tissue biopsies with a diameter of $1 \mathrm{~mm}$ from the specific areas of the 'donor' block. The tissue core biopsies were transferred to the recipient paraffin block at defined array positions. The tissue microarrays contained tissue samples from 45 formalin-fixed paraffin-embedded cancer specimens with known diagnosis, and the corresponding ANCTs from these patients. The block was incubated in an oven at $45^{\circ} \mathrm{C}$ for 20 min to allow complete embedding of the grafted tissue cylinders in the paraffin of the recipient block, and then stored at $4^{\circ} \mathrm{C}$ until microtome sectioning.

Immunohistochemical staining. Tissue microarray sections were processed for IHC analysis of TNFAIP1 protein as follows. IHC examinations were carried out on 3-mm thick sections. For anti-TNFAIP1 IHC, unmasking was performed with $10 \mathrm{mM}$ sodium citrate buffer, $\mathrm{pH} 6.0$, at $90^{\circ} \mathrm{C}$ for $30 \mathrm{~min}$. For anti-TNFAIP1 IHC, antigen unmasking was not necessary. Sections were incubated in $0.03 \%$ hydrogen peroxide for $10 \mathrm{~min}$ at room temperature to remove endogenous peroxidase activity, and then in blocking serum $[0.04 \%$ bovine serum albumin (A2153; Sigma-Aldrich, Shanghai, China) and $0.5 \%$ normal goat serum (X0907; Dako Corporation, Carpinteria, CA, USA) in phosphate-buffered saline (PBS)] for $30 \mathrm{~min}$ at room temperature. The anti-TNFAIP1 antibody was used at a dilution of 1:200. The antibody was incubated overnight at $4^{\circ} \mathrm{C}$. Sections were then washed three times for $5 \mathrm{~min}$ in PBS. Non-specific staining was blocked with $0.5 \%$ casein and $5 \%$ normal serum for $30 \mathrm{~min}$ at room temperature. Finally, staining was developed using diaminobenzidine substrate, and sections were counterstained with hematoxylin. Normal serum or PBS was used to replace the anti-TNFAIP1 antibody in the negative controls.

Quantification of protein expression. The expression of TNFAIP1 was semi-quantitatively estimated as total immunostaining scores, which were calculated as the product of a proportion score and an intensity score. The proportion and intensity of the staining were evaluated independently by two observers. The proportion score reflected the fraction of positive staining cells ( 0 , none; $1,<10 \% ; 2,10$ to $\geq 25 \% ; 3,>25$ to $50 \% ; 4,>50 \%$ ), and the intensity score represented the staining intensity ( 0 , no staining; 1 , weak; 2 , intermediate; 3 , strong). Finally, a total expression score was given ranging from 0 to 12 . Based on the analysis in advance, TNFAIP1 expression was categorized into two groups: low-level TNFAIP1 expression (score 0-3) and high-level TNFAIP1 expression (score 4-12). The scoring was independently assessed by two pathologists.

Cell culture and transfection. OS cell lines were cultured in DMEM supplemented with $10 \%$ heat-inactivated FBS, $100 \mathrm{U} /$ $\mathrm{ml}$ of penicillin and $100 \mu \mathrm{g} / \mathrm{ml}$ of streptomycin. Cells in this medium were placed in a humidified atmosphere containing $5 \% \mathrm{CO}_{2}$ at $37^{\circ} \mathrm{C}$. Cells were subcultured at a 1:5 dilution in medium containing $300 \mu \mathrm{g} / \mathrm{ml} \mathrm{G} 418$ (an aminoglycoside antibody, commonly used as a stable transfection reagent in molecular genetic testing). On the day of transduction, OS cells were replated at $5 \times 10^{4}$ cells/well in 24 -well plates containing serum-free growth medium with Polybrene $(5 \mathrm{mg} / \mathrm{ml})$. When reaching 50\% confluency, the cells were transfected with the recombinant experimental or control virus at the optimal multiplicity of infection (MOI) of 50 , and cultured at $37^{\circ} \mathrm{C}$ in $5 \% \mathrm{CO}_{2}$ for $4 \mathrm{~h}$. Then the supernatant was discarded and serum containing growth medium was added. At 4 days posttransduction, transduction efficiency was measured by the frequency of green fluorescent protein (GFP)-positive cells. Positive and stable transfectants were selected and expanded for further study. The TNFAIP1 siRNA (siTNFAIP1) vectorinfected clone, the negative control vector-infected cells and the untreated cells were designated as the Lv-siTNFAIP1 group, NC group and CON group, respectively.

Quantitative real-time PCR. To quantitatively determine the mRNA expression level of TNFAIP1 in OS cells, real-time 
PCR was performed. Total RNA was extracted from each clone using TRIzol according to the manufacturer's protocol. Reverse transcription was carried out using M-MLV, and cDNA amplification was performed using the SYBR-Green Master Mix kit according to the manufacturer's guidelines. The TNFAIP1 gene was amplified using a specific oligonucleotide primer, and the $\beta$-actin gene was used as an endogenous control. Data were analyzed using the comparative $\mathrm{Ct}$ method $\left(2^{-\Delta \Delta \mathrm{Ct}}\right)$. Three separate experiments were performed for each clone.

Western blot assay. OS cell lines were harvested and extracted using lysis buffer (Tris-HCl, SDS, mercaptoethanol and glycerol). Cell extracts were boiled for $5 \mathrm{~min}$ in loading buffer, and then an equal amount of cell extracts was separated on $15 \%$ SDS-PAGE gels. Separated protein bands were transferred onto polyvinylidene fluoride (PVDF) membranes, which were subsequently blocked in 5\% skim milk powder. Primary antibodies against TNFAIP1, p65NF- $\kappa$, PCNA, matrix metalloproteinase-2 (MMP-2) and caspase-3 were diluted according to the manufacturer's instructions and incubated overnight at $4^{\circ} \mathrm{C}$. Subsequently, horseradish peroxidase-linked secondary antibodies were added at a dilution of 1:1,000 and incubated at room temperature for $2 \mathrm{~h}$. The membranes were washed 3 times with PBS, and the immunoreactive bands were visualized using the ECL Plus kit according to the manufacturer's instructions. The relative protein levels in the different cell lines were normalized to the concentration of $\beta$-actin. Three separate experiments were performed for each clone.

Cell proliferation assay. Cell proliferation was analyzed using the MTT assay. Briefly, cells infected with the siTNFAIP1 virus were incubated in 96-well-plates at a density of $1 \times 10^{5}$ cells/well with DMEM supplemented with $10 \%$ FBS. Cells were treated with $20 \mu \mathrm{l}$ of MTT dye at $0,24,48$ and $72 \mathrm{~h}$, and subsequently incubated with $150 \mu \mathrm{l}$ of DMSO for $5 \mathrm{~min}$. The color reaction was measured at $570 \mathrm{~nm}$ using an Enzyme Immunoassay Analyzer (Bio-Rad, Hercules, CA, USA). The proliferation activity was calculated for each clone.

Transwell invasion assay. Transwell filters were coated with Matrigel $(3.9 \mu \mathrm{g} / \mu \mathrm{l} ; 60-80 \mu \mathrm{l})$ on the upper surface of a polycarbonate membrane (diameter, $6.5 \mathrm{~mm}$; pore size, $8-\mu \mathrm{m})$. After incubation at $37^{\circ} \mathrm{C}$ for $30 \mathrm{~min}$, the Matrigel solidified and served as the extracellular matrix for analysis of tumor cell invasion. Harvested cells $\left(1 \times 10^{5}\right)$ in $100 \mu 1$ of serum-free DMEM were added into the upper compartment of the chamber. A total of $200 \mu \mathrm{l}$ of conditioned medium derived from NIH3T3 cells was used as a source of chemoattractant, which was placed in the bottom compartment of the chamber. After $24 \mathrm{~h}$ of incubation at $37^{\circ} \mathrm{C}$ with $5 \% \mathrm{CO}_{2}$, the medium was removed from the upper chamber. The non-invaded cells on the upper side of the chamber were scraped off with a cotton swab. Cells that had migrated from the Matrigel into the pores of the inserted filter were fixed with $100 \%$ methanol, stained with hematoxylin, then mounted and dried at $80^{\circ} \mathrm{C}$ for $30 \mathrm{~min}$. The number of cells invading through the Matrigel was counted in 3 randomly selected visual fields from the central and peripheral portion of the filter by using an inverted microscope (x200 magnification). Each assay was repeated 3 times.

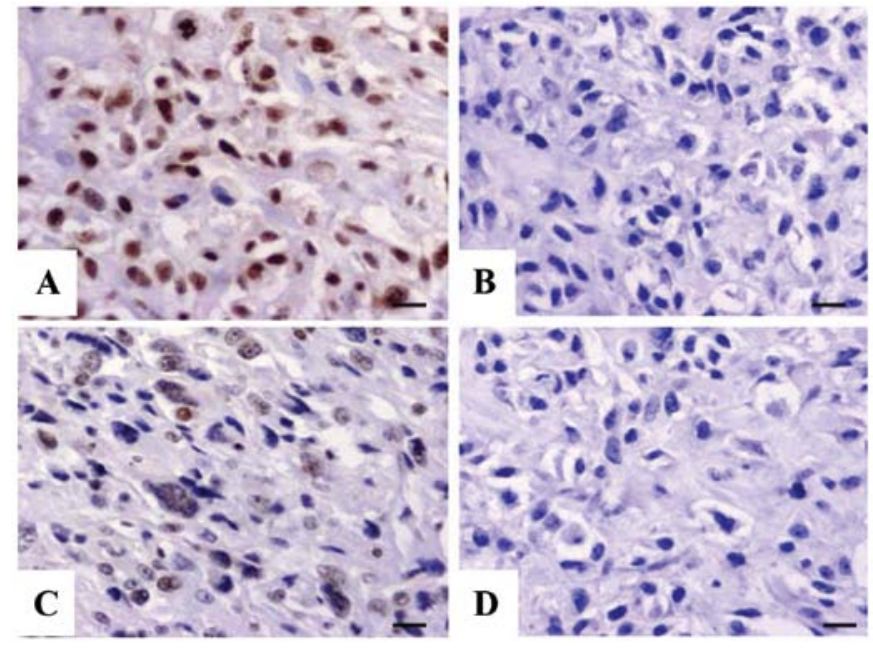

Figure 1. Expression of TNFAIP1 protein in OS tissues (magnification, $\mathrm{x} 400$ ) OS tissues were immunohistochemically stained with an anti-TNFAIP antibody. The positive expression of TNFAIP, mainly localized in the nucleus, was increased in the OS tissues compared with the ANCTs. (A) Positive expression in OS tissues. (B) Negative expression in OS tissues. (C) Positive expression in ANCTs. (D) Negative expression in ANCTs. TNFAIP1, tumor necrosis factor- $\alpha$-inducible protein-1; OS, osteosarcoma; ANCTs, adjacent non-cancerous tissues.

Analysis of cell apoptosis. To detect cell apoptosis, OS cells treated with siTNFAIP1 were trypsinized, washed with cold PBS and resuspended in binding buffer according to the instructions provided in the apoptosis kit. FITC-Annexin V and PI were added to the fixed cells for $20 \mathrm{~min}$ in darkness at room temperature. Then, Annexin V binding buffer was added to the mixture before the fluorescence was measured on the FACSort flow cytometer. Cell apoptosis was analyzed using CellQuest software (Becton-Dickinson, USA). Three separate experiments were performed for each clone.

Statistical analysis. SPSS 20.0 was used for statistical analysis. Kruskal-Wallis $\mathrm{H}$ and Chi-square tests were used to analyze the expression rate in all groups. One-way analysis of variance (ANOVA) was used to analyze the differences between groups. The LSD method of multiple comparisons was used when the probability for ANOVA was statistically significant. Statistical significance was set at $\mathrm{P}<0.05$.

\section{Results}

Expression of TNFAIP1 in OS tissues. The expression of TNFAIP1 protein was evaluated using IHC staining in OS tissues. Different levels of positive expression of TNFAIP1 protein were detected in the OS tissues (Fig. 1A and B) and the ANCTs (Fig. 1C and D). Positive TNFAIP1 immunostaining was mainly localized in the nucleus of the OS tissue cells. According to the TNFAIP1 immunoreactive intensity, the positive expression of TNFAIP1 in the OS tissues was significantly increased compared with that in the ANCTs $(\mathrm{P}=0.018)$ (Table II).

Correlation of TNFAIP1 expression with clinicopathological characteristics. The correlation of TNFAIP1 expression with various clinicopathologic factors was analyzed. As 
Table I. Correlation of TNFAIP1 expression and clinicopathological factors of the OS patients.

\begin{tabular}{|c|c|c|c|c|}
\hline \multirow[b]{2}{*}{ Variables } & \multirow[b]{2}{*}{ Cases (n) } & \multicolumn{2}{|c|}{$\begin{array}{c}\text { TNFAIP1 } \\
\text { expression (n) }\end{array}$} & \multirow[b]{2}{*}{ P-value } \\
\hline & & - & + & \\
\hline Total & 45 & 12 & 33 & \\
\hline Age (years) & & & & 0.714 \\
\hline$<20$ & 28 & 8 & 20 & \\
\hline$\geq 20$ & 17 & 4 & 13 & \\
\hline Gender & & & & 0.964 \\
\hline Male & 26 & 7 & 19 & \\
\hline Female & 19 & 5 & 14 & \\
\hline Histology & & & & 0.641 \\
\hline Osteoblastic & 21 & 7 & 14 & \\
\hline Chondroblastic & 15 & 3 & 12 & \\
\hline Fibroblastic & 9 & 2 & 7 & \\
\hline Ennecking staging & & & & 0.492 \\
\hline I & 13 & 3 & 10 & \\
\hline II & 24 & 8 & 16 & \\
\hline III & 8 & 1 & 7 & \\
\hline Distant metastasis & & & & 0.029 \\
\hline No & 18 & 8 & 10 & \\
\hline Yes & 27 & 4 & 23 & \\
\hline
\end{tabular}

TNFAIP1, tumor necrosis factor- $\alpha$-inducible protein-1; OS, osteosarcoma.

shown in Table I, increased expression of TNFAIP1 was closely correlated with the distant metastasis of OS patients $(\mathrm{P}=0.029)$. However, no significant association was found between TNFAIP1 expression and other factors including age, gender of the patients, and histology and Ennecking stage of the tumor $(\mathrm{P}>0.05$, respectively).

Effect of TNFAIPI knockdown on the expression of NF- $\kappa B$. Lentiviruses of different multiplicity of infection (MOI) were transfect into OS cells (MG-63 and U-2 OS), and the transfection efficiency of siTNFAIP1 (MOI=50) reached $>70 \%$. After siTNFAIP1 was transfected into the OS cells for $24 \mathrm{~h}$, the expression levels of TNFAIP1 mRNA (Fig. 2A and B) and
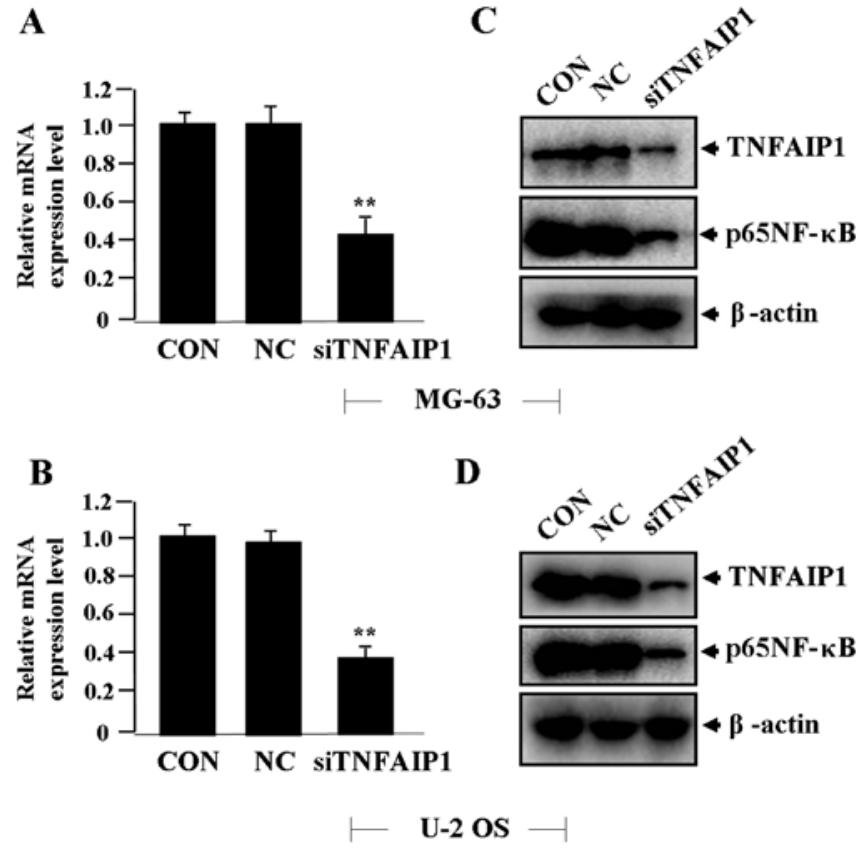

Figure 2. Effect of TNFAIP1 on the expression of NF- $\mathrm{BB}$. After OS cells were transfected with siTNFAIP1 for $24 \mathrm{~h}$, the expression levels of TNFAIP1 and p65NF- $\mathrm{kB}$ were detected by real-time PCR (A and B) and western blot assays (C and D), indicating the decreased expression of TNFAIP1 and p65NF- $\mathrm{kB}$ in the siTNFAIP1 group compared with the levels in the NC and CON groups (each ${ }^{* *} \mathrm{P}<0.01$ ). TNFAIP1, tumor necrosis factor- $\alpha$-inducible protein-1; NF- $\kappa B$, nuclear factor- $\kappa \mathrm{B}$; OS, osteosarcoma; siTNFAIP1, TNFAIP1 siRNA; NC, negative control.

protein levels of TNFAIP1 and p65NF-kB (Fig. 2C and D) were detected by real-time PCR and western blotting, indicating decreased expression of TNFAIP1 and p65NF- $\kappa \mathrm{B}$ in the siTNFAIP1 group compared with the NC and CON groups.

Effect of TNFAIP1 knockdown on cell proliferation. Deregulated cell proliferation is a hallmark of cancer. To confirm the effect of TNFAIP1 on OS cell growth, we examined cell proliferative activities by MTT assay. The results showed that knockdown of TNFAIP1 diminished the proliferative activities of the OS cells in a time-dependent manner compared to the NC group (Fig. 3A and B). In addition, the expression level of PCNA protein, examined by western blotting (Fig. 3C and D), was found to be significantly downregulated in the siTNFAIP1 group when compared with the level in the $\mathrm{NC}$ and $\mathrm{NC}$ groups.

Table II. Expression of TNFAIP1 protein in OS tissues.

\begin{tabular}{llllllllll}
\hline & & \multicolumn{3}{c}{ TNFAIP1 expression (n) } & & Positive \\
\cline { 3 - 6 } Target & Sample & - & + & ++ & +++ & Total & rate (\%) & $\chi^{2}$ & P-value \\
\hline \multirow{2}{*}{ TNFAIP1 } & OS & 12 & 17 & 10 & 6 & 45 & 73.3 & & 0.018 \\
& ANCTs & 23 & 13 & 6 & 3 & 45 & 48.9 & 5.607 &
\end{tabular}

TNFAIP1, tumor necrosis factor- $\alpha$-inducible protein-1; OS, osteosarcoma; ANCTs, adjacent non-cancer tissues. 


\section{A}

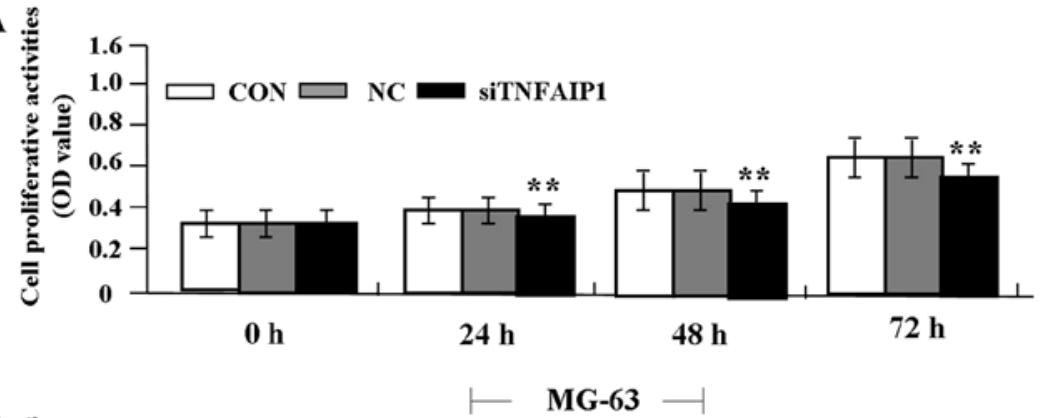

$B$

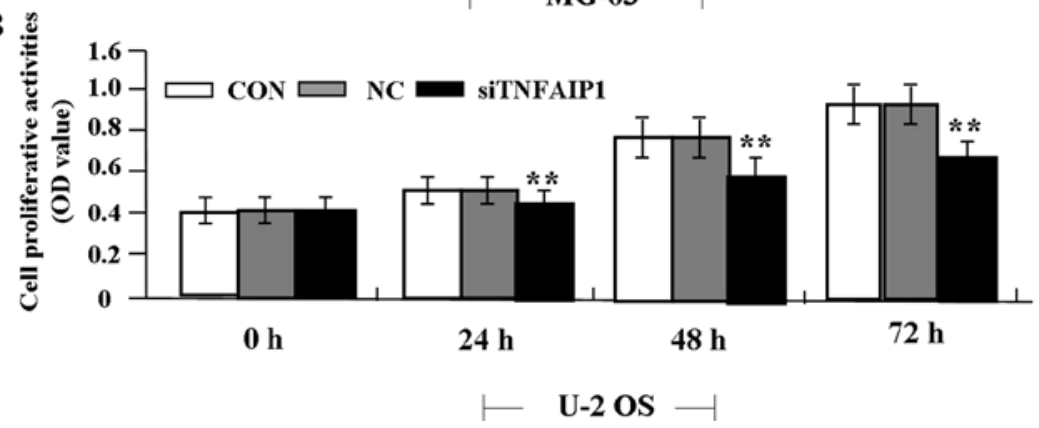

C

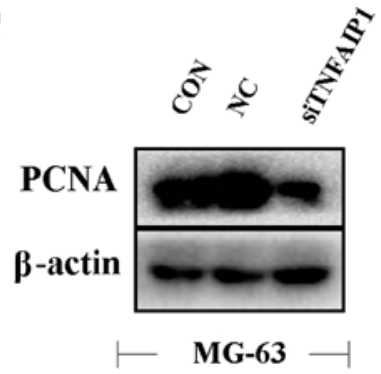

D

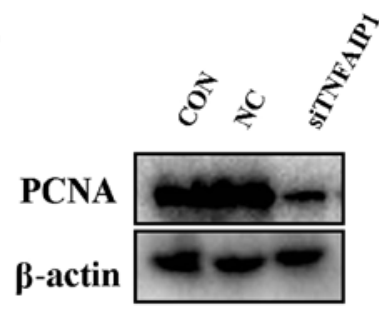

$\mathrm{U}-2$ OS

Figure 3. Effect of TNFAIP1 on cell proliferation. (A and B) MTT assay was used to examine the cell proliferative activity, indicating that cell proliferation was significantly suppressed in a time-dependent manner in the siTNFAIP1 group compared with the cell proliferative activity of the NC and CON groups $\left({ }^{* *} \mathrm{P}<0.01\right)$. (C and D) The expression level of PCNA protein examined by western blotting was downregulated in the siTNFAIP1 group when compared with the level in the NC and CON groups. TNFAIP1, tumor necrosis factor- $\alpha$-inducible protein-1; siTNFAIP1, TNFAIP1 siRNA; NC, negative control; PCNA, proliferating cell nuclear antigen.

$\mathbf{A}$
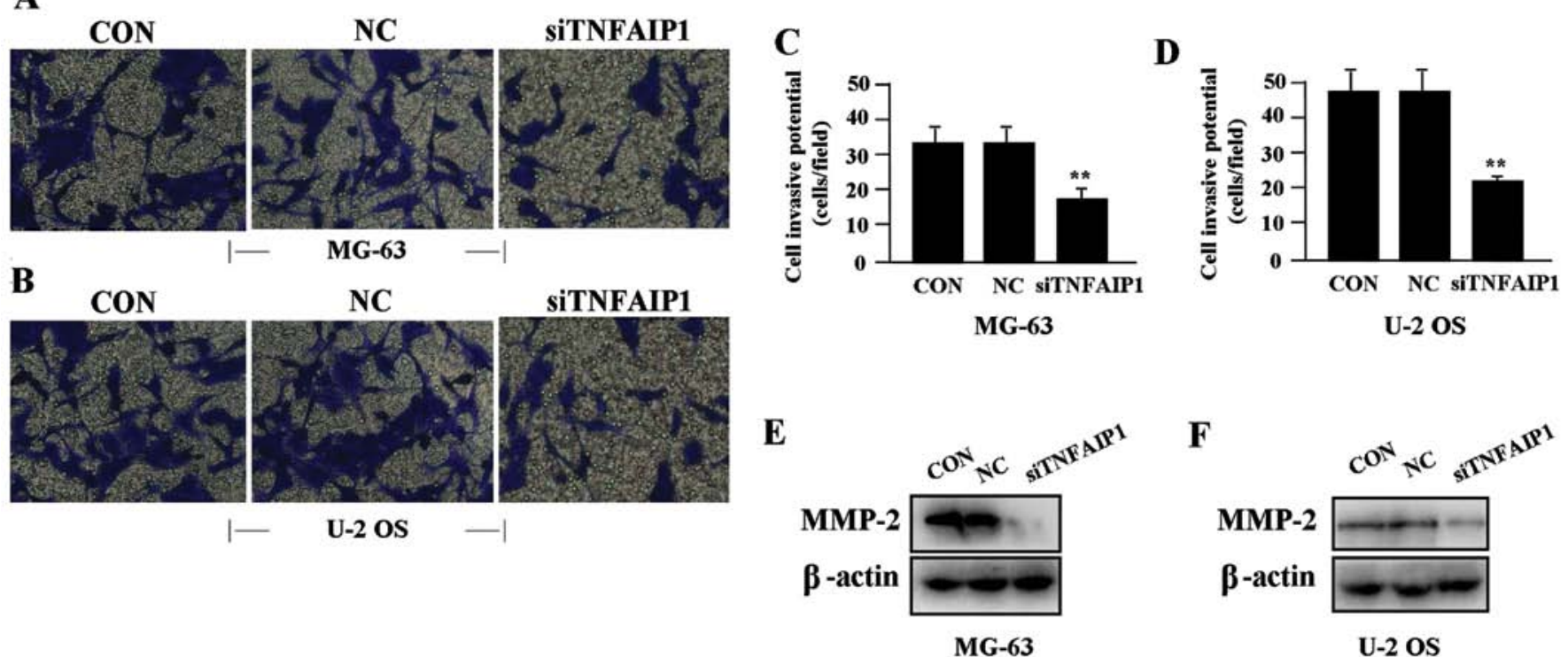

Figure 4. Effect of TNFAIP1 on cell invasion. (A-D) Transwell assay was performed to determine cell invasion. Cell invasive potential was lower in the siTNFAIP1 group when compared with the invasive potential of the $\mathrm{NC}$ and $\mathrm{CON}$ groups ( $\left({ }^{* *} \mathrm{P}<0.01\right)$. (E and $\left.\mathrm{F}\right)$ The expression level of MMP-2 protein examined by western blotting was downregulated in the siTNFAIP1 group when compared with the level in the NC and CON groups. TNFAIP1, tumor necrosis factor- $\alpha$-inducible protein-1; siTNFAIP1, TNFAIP1 siRNA; NC, negative control; MMP-2, matrix metalloproteinase-2.

Effect of TNFAIPI knockdown on cell invasion. To determine the effect of TNFAIP1 on cell invasion, a Transwell assay was performed. The invasive potential of tumor cells in the Transwell assay was determined by the ability of cells to invade a matrix barrier containing laminin and type IV collagen, the major components of the basement membrane. Representative micrographs of Transwell filters are shown in Fig. 4A and B. We found that the invasive potential of OS cells was markedly lowered in the siTNFAIP1 group when compared to the invasive potential in the $\mathrm{NC}$ and $\mathrm{CON}$ groups $(\mathrm{P}<0.01)$ (Fig. 4C and D). In addition, the expression level of MMP-2 protein, examined by western blotting (Fig. 4E and F), was found to be significantly downregulated in the siTNFAIP1 group when compared to the level in the $\mathrm{NC}$ and $\mathrm{CON}$ groups. 
A

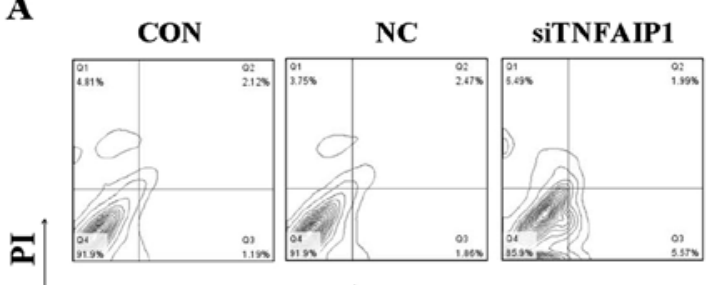

Annexin V (MG-63)

$\mathbf{B}$

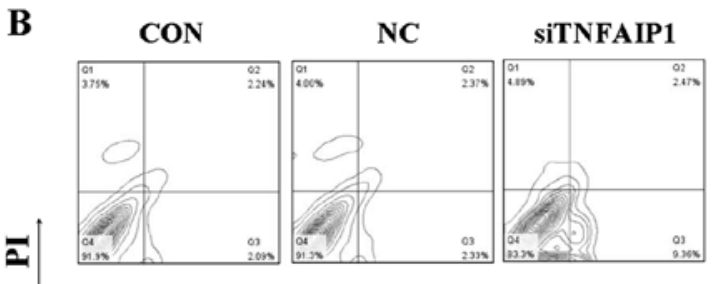

Annexin V (U-2 OS)

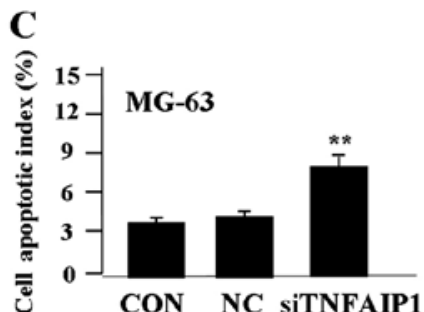

D

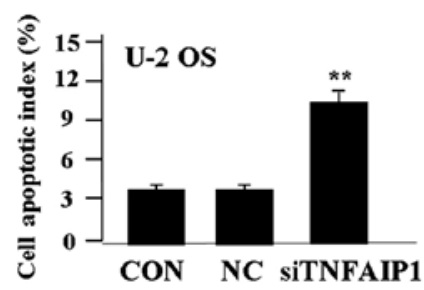

$\mathbf{E}$

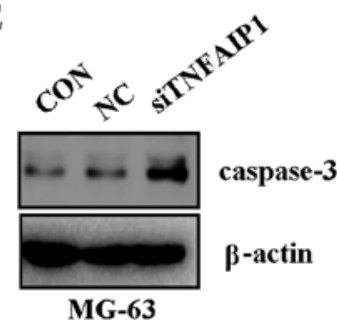

F

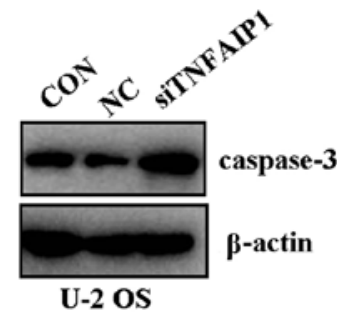

Figure 5. Effect of TNFAIP1 on cell apoptosis. (A-D) Flow cytometric analysis was conducted to determine cell apoptosis. Cell apoptotic indices were much higher in the siTNFAIP1 group when compared to the indices in the NC and CON groups $\left({ }^{* *} \mathrm{P}<0.01\right)$. (E and F) The expression level of caspase-3 protein as examined by western blotting was upregulated in the siTNFAIP1 group when compared with the level in the NC and CON groups. TNFAIP1, tumor necrosis factor- $\alpha$-inducible protein-1; siTNFAIP1, TNFAIP1 siRNA; NC, negative control.

Effect of TNFAIPl knockdown on cell apoptosis. To determine whether TNFAIP1 affects OS cell apoptosis, flow cytometric analysis with PI/FITC-Annexin V staining was performed. We found that the apoptosis indices of the OS cells were significantly higher in the siTNFAIP1 group when compared to the indices in the NC and CON groups (Fig. 5A-D). Additionally, to determine whether TNFAIP1 regulates the expression of caspase- 3 protein, western blotting was carried out. The protein level of caspase-3 was found to be upregulated in the siTNFAIP1 group in comparison with the level in the $\mathrm{NC}$ and CON groups (Fig. 5E and F).

\section{Discussion}

TNF- $\alpha$ is an inflammatory cytokine that is present in the microenvironment of many types of tumors, and is known to promote tumor progression. Overexpression of TNFAIP1 or TNFAIP2 correlates with tumor invasion and metastasis, and serves as an independent prognostic indicator for breast cancer (9) and nasopharyngeal carcinoma (15). The TNFAIP2 miRNA binding site (s8126 T>C SNP) is also correlated with a significantly elevated risk of gastric cancer, suggesting a marker for susceptibility to gastric cancer (16). To verify the correlation of TNFAIP1 expression with OS, we examined the expression of TNFAIP1 in human OS tissues. The present study showed that TNFAIP1 was markedly upregulated in the nuclei of OS tissue cells when compared to the ANCTs, and was positively correlated with the distant metastasis of OS patients. These studies demonstrated that TNFAIP1 may serve as a potential biomarker involved in the development of OS.

Moreover, the function of TNFAIP requires further research in cancer. First, TNFAIP1 confers acquired resistance to paclitaxel, while knockdown of TNFAIP1 was found to increase the tumor response to paclitaxel (17), suggesting that TNFAIP may represent a valuable therapeutic target for the treatment of cancer. Furthermore, TNFAIP1 was found to be downregulated by miR-372/-373 and participates in cell apoptosis and proliferation via the NF- $\kappa \mathrm{B}$ pathway $(18,19)$. In addition, TNFAIP2 was found to promote tumor progression in nasopharyngeal carcinoma (NPC) (20), while knockdown of TNFAIP2 was found to markedly reduce the migration and invasion of NPC cells (15). The present study also showed that knockdown of TNFAIP1 repressed cell proliferation and invasion, and induced cell apoptosis in OS cells, which is supported by data that TNFAIP2 can mediate the inhibitory effects of retinoic acid on endometrial cancer cell growth, invasion and apoptosis escape (21), suggesting TNFAIP1/2 as a potential target for retinoic acid in acute promyelocytic leukemia (22). Yet, research found that simultaneous expression of RhoB and TNFAIP1 resulted in a marked increase in apoptosis in HeLa cells (23), which is opposite from our findings concerning the decrease in cell apoptosis induced by TNFAIP1 in OS cells, suggesting that the underlying molecular mechanisms of TNFAIP1 in cancer require further exploration.

Activation of $\mathrm{NF}-\kappa \mathrm{B}$ promotes the development of OS (14), while inhibition of $\mathrm{NF}-\kappa \mathrm{B}$ activity suppresses the tumorigenicity of Ewing sarcoma EW7 cells (24). It has been reported that $\mathrm{NF}-\kappa \mathrm{B}-$ mediated transcriptional upregulation of TNFAIP2 by the Epstein-Barr virus oncoprotein, LMP1, promotes cell motility in NPC (20). However, few studies have shown that TNFAIP1 inhibits the transcriptional activities of $\mathrm{NF}-\kappa \mathrm{B}(25)$. In the present study, we found that knockdown of TNFAIP1 downregulated the expression of NF- $\kappa \mathrm{B}$ at the translational level in OS cells, suggesting that TNFAIP1 may promote the progression of OS through activation of the $\mathrm{NF}-\kappa \mathrm{B}$ pathway.

PCNA is a nuclear protein that is expressed in proliferating cells and may be required for maintaining cell proliferation, and is used as a marker for cell proliferation of colon cancer (26). MMP-2 is believed to be a key enzyme involved in the degradation of type IV collagen, and a high level of MMP-9 in tissues is associated with tumor invasion and metastasis (27). NF- $\kappa \mathrm{B}$ was found to promote the growth and invasion and block the apoptosis of tumor cells via regulation of 
the expression of PCNA (28), MMP-2 (29) and apoptosis (30). Moreover, it was found that TNFAIP1 promotes cell growth and gene amplification in a PCNA-dependent way $(5,31)$, yet no report has demonstrated the regulation of the expression of MMP-2 and caspase-3 by TNFAIP1. In the present study, we found that knockdown of TNFAIP1 downregulated the expression of PCNA and MMP-9 and upregulated the expression of caspase-3 in OS cells, suggesting that TNFAIP1 may promote the progression of OS through $\mathrm{NF}-\kappa \mathrm{B}$ pathway-mediated regulation of PCNA, MMP-2 and caspase-3 expression.

In conclusion, our findings indicate that high expression of TNFAIP1 is associated with the distant metastasis of OS, and knockdown of TNFAIP1 inhibits the growth and invasion, and induces apoptosis in OS cells through inhibition of the NF- $\kappa \mathrm{B}$ pathway, suggesting that TNFAIP1 may act as a potential therapeutic target for the treatment of cancer.

\section{References}

1. Kuijjer ML, van den Akker BE, Hilhorst R, et al: Kinome and mRNA expression profiling of high-grade osteosarcoma cell lines implies Akt signaling as possible target for therapy. BMC Med Gen 7: 4, 2014.

2. Buddingh EP, Anninga JK, Versteegh MI, et al: Prognostic factors in pulmonary metastasized high-grade osteosarcoma. Pediatr Blood Cancer 54: 216-221, 2010.

3. Bacci G, Longhi A, Versari M, et al: Prognostic factors for osteosarcoma of the extremity treated with neoadjuvant chemotherapy: 15-year experience in 789 patients treated at a single institution. Cancer 106: 1154-1161, 2006.

4. Savage SA, Mirabello L, Wang Z, et al: Genome-wide association study identifies two susceptibility loci for osteosarcoma. Nat Genet 45: 799-803, 2013.

5. Yang L, Liu N, Hu X, et al: CK2 phosphorylates TNFAIP1 to affect its subcellular localization and interaction with PCNA Mol Biol Rep 37: 2967-2973, 2010

6. Link CD, Taft A, Kapulkin V, et al: Gene expression analysis in a transgenic Caenorhabditis elegans Alzheimer's disease model Neurobiol Aging 24: 397-413, 2003.

7. Lin MC, Lee NP, Zheng N, et al: Tumor necrosis factor- $\alpha$ induced protein 1 and immunity to hepatitis B virus. World J Gastroenterol 11: 7564-7568, 2005.

8. Gupta J, Gaikwad AB and Tikoo K: Hepatic expression profiling shows involvement of PKC epsilon, DGK eta, Tnfalpha, and Rho kinase in type 2 diabetic nephropathy rats. J Cell Biochem 111: 944-954, 2010

9. Grinchuk OV, Motakis E and Kuznetsov VA: Complex senseantisense architecture of TNFAIP1/POLDIP2 on 17q11.2 represents a novel transcriptional structural-functional gene module involved in breast cancer progression. BMC Genomics 11 (Suppl 1): S9, 2010.

10. Kondratiev S, Duraisamy S, Unitt CL, et al: Aberrant expression of the dendritic cell marker TNFAIP2 by the malignant cells of Hodgkin lymphoma and primary mediastinal large B-cell lymphoma distinguishes these tumor types from morphologically and phenotypically similar lymphomas. Am J Surg Pathol 35: 1531-1539, 2011.

11. Dumon K, Rossbach C, Harms B, et al: Tumor necrosis factoralpha (TNF-alpha) gene polymorphism in surgical intensive care patients with SIRS. Langenbecks Arch Chir Suppl Kongressbd 115 (Suppl I): 387-390, 1998 (In German).

12. Liu XW, Lu FG, Zhang GS, et al: Proteomics to display tissue repair opposing injury response to LPS-induced liver injury. World J Gastroenterol 10: 2701-2705, 2004.
13. Sparks AB, Peterson SN, Bell C, et al: Mutation screening of the TNFRSF11A gene encoding receptor activator of $N F \kappa B$ (RANK) in familial and sporadic Paget's disease of bone and osteosarcoma. Calcif Tissue Int 68: 151-155, 2001.

14. Javelaud D and Besançon F: NF- $\kappa B$ activation results in rapid inactivation of JNK in TNF $\alpha$-treated Ewing sarcoma cells: a mechanism for the anti-apoptotic effect of NF- $\kappa$ B. Oncogene 20: 4365-4372, 2001.

15. Chen LC, Chen CC, Liang Y, et al: A novel role for TNFAIP2: its correlation with invasion and metastasis in nasopharyngeal carcinoma. Mod Pathol 24: 175-184, 2011.

16. Xu Y, Ma H, Yu H, et al: The miR-184 binding-site rs8126 T>C polymorphism in TNFAIP2 is associated with risk of gastric cancer. PLoS One 8: e64973, 2013.

17. Zhu Y, Yao Z, Wu Z, et al: Role of tumor necrosis factor alphainduced protein 1 in paclitaxel resistance. Oncogene: Aug 5, 2013 (Epub ahead of print). doi: 10.1038/onc.2013.299.

18. Zhou C,Li X,Zhang X, et al: microRNA-372 maintains oncogene characteristics by targeting TNFAIP1 and affects NFKB signaling in human gastric carcinoma cells. Int J Oncol 42: 635-642, 2013

19. Zhang X, Li X, Tan Z, et al: MicroRNA-373 is upregulated and targets TNFAIP1 in human gastric cancer, contributing to tumorigenesis. Oncol Lett 6: 1427-1434, 2013.

20. Chen CC, Liu HP, Chao M, et al: NF- $\kappa \mathrm{B}-$ mediated transcriptional upregulation of TNFAIP2 by the Epstein-Barr virus oncoprotein, LMP1, promotes cell motility in nasopharyngeal carcinoma. Oncogene: Aug 26, 2013 (Epub ahead of print). doi: 10.1038/onc.2013.345.

21. Cheng YH, Utsunomiya H, Pavone ME, et al: Retinoic acid inhibits endometrial cancer cell growth via multiple genomic mechanisms. J Mol Endocrinol 46: 139-153, 2011.

22. Rusiniak ME, Yu M, Ross DT, et al: Identification of B94 (TNFAIP2) as a potential retinoic acid target gene in acute promyelocytic leukemia. Cancer Res 60: 1824-1829, 2000.

23. Kim DM, Chung KS, Choi SJ, et al: RhoB induces apoptosis via direct interaction with TNFAIP1 in HeLa cells. Int J Cancer 125: 2520-2527, 2009.

24. Javelaud D, Poupon MF, Wietzerbin and Besançon F: Inhibition of constitutive NF- $\mathrm{KB}$ activity suppresses tumorigenicity of Ewing sarcoma EW7 cells. Int J Cancer 98: 193-198, 2002.

25. Hu X, Yan F, Wang F, et al: TNFAIP1 interacts with KCTD10 to promote the degradation of KCTD10 proteins and inhibit the transcriptional activities of NF- $\mathrm{BB}$ and AP-1. Mol Biol Rep 39: 9911-9919, 2012.

26. Risio M: Cell proliferation in colorectal tumor progression: an immunohistochemical approach to intermediate biomarkers. J Cell Biochem (Suppl) 16G: 79-87, 1992.

27. Hornebeck W, Lambert E, Petitfrère E and Bernard P: Beneficial and detrimental influences of tissue inhibitor of metalloproteinase-1 (TIMP-1) in tumor progression. Biochimie 87: 377-383, 2005.

28. Shah SJ and Sylvester PW: $\gamma$-Tocotrienol inhibits neoplastic mammary epithelial cell proliferation by decreasing Akt and nuclear factor $\kappa$ B activity. Exp Biol Med 230: 235-241, 2005.

29. Philip S, Bulbule A and Kundu GC: Osteopontin stimulates tumor growth and activation of promatrix metalloproteinase-2 through nuclear factor- $\kappa \mathrm{B}$-mediated induction of membrane type 1 matrix metalloproteinase in murine melanoma cells. J Biol Chem 276: 44926-44935, 2001.

30. Moalic-Juge S, Liagre B, Duval R, et al: The anti-apoptotic property of NS-398 at high dose can be mediated in part through NF- $\kappa \mathrm{B}$ activation, hsp70 induction and a decrease in caspase-3 activity in human osteosarcoma cells. Int J Oncol 20: 1255-1262, 2002.

31. Zhou J, Hu X, Xiong X, et al: Cloning of two rat PDIP1 related genes and their interactions with proliferating cell nuclear antigen. J Exp Zool A Comp Exp Biol 303: 227-240, 2005. 